\title{
Suppression and enhancement of impurity scattering in a Bose-Einstein condensate
}

\author{
A.P. Chikkatur, A. Görlitz, D.M. Stamper-Kurn,* S. Inouye, S. Gupta, and W. Ketterle \\ Department of Physics and Research Laboratory of Electronics, \\ Massachusetts Institute of Technology, Cambridge, MA 02139
}

(October 28, 2018)

\begin{abstract}
Impurity atoms propagating at variable velocities through a trapped Bose-Einstein condensate were produced using a stimulated Raman transition. The redistribution of momentum by collisions between the impurity atoms and the stationary condensate was observed in a time-of-flight analysis. The collisional cross section was dramatically reduced when the velocity of the impurities was reduced below the speed of sound of the condensate, in agreement with the Landau criterion for superfluidity. For large numbers of impurity atoms, we observed an enhancement of atomic collisions due to bosonic stimulation. This enhancement is analogous to optical superradiance.
\end{abstract}

One manifestation of superfluidity is that objects traveling below a critical velocity $v_{L}$ through a superfluid propagate without dissipation. Landau [1] used simple kinematic arguments to derive an expression for the critical velocity $v_{L}=\min (E(p) / p)$, where $E(p)$ is the energy of an elementary excitation with momentum $p$.

When superfluid ${ }^{4} \mathrm{He}$ was forced through capillaries, adsorbed films and tightly packed powders [2], the onset of dissipation was found at velocities much lower than the Landau critical velocity due to turbulence and vortex formation in the superfluid. The Landau critical velocity can usually only be observed by moving microscopic particles through the superfluid which do not create a macroscopic flow pattern. Studies of superfluidity with microscopic objects were pursued in liquid ${ }^{4} \mathrm{He}$ by dragging negative ions through pressurized ${ }^{4} \mathrm{He}$, 3, th, and by scattering ${ }^{3} \mathrm{He}$ atoms off superfluid ${ }^{4} \mathrm{He}$ droplets $[5$.

Atomic Bose-Einstein condensates are superfluid gases and show phenomena analogous to superfluid liquids, albeit at eight orders of magnitude lower density. For a homogeneous gaseous Bose-Einstein condensate, the Bogoliubov spectrum indicates a Landau critical velocity equal to the speed of sound $v_{L}=c \equiv \sqrt{\mu / M}$, where $\mu$ is the chemical potential and $M$ is the mass of condensate atoms. The first evidence for a critical velocity in a Bose condensate was obtained by stirring the condensate with a macroscopic object (a laser beam) [6]. The observed critical velocity was about a factor of four smaller than the Bogoliubov speed of sound. Recent studies of superfluidity have revealed quantized vortices [7,8] and a non-classical moment of inertia [9].

In this Letter, we report on a study of the motion of microscopic impurities through a gaseous Bose-Einstein condensate. The impurity atoms were created using a stimulated Raman process which transferred a small fraction of the condensate atoms into an untrapped hyperfine state with well-defined initial velocity. As these impurities traversed the condensate, they dissipated energy by colliding with the stationary condensate, which re- sulted in a redistribution of momenta of the impurities. As the impurity velocity was reduced below the speed of sound, we observed a dramatic reduction in the probability of collisions, which is evidence for superfluidity in Bose-Einstein condensates.

Our experiments were performed on Bose-Einstein condensates of sodium atoms in the $\left|F=1, m_{F}=-1\right\rangle$ hyperfine ground state. Condensates of $\sim 10^{7}$ atoms were created using laser and evaporative cooling and stored in a cylindrically symmetric magnetic trap 10 with an axial trapping frequency of $16 \mathrm{~Hz}$. By adiabatically changing the radial trapping frequency between $165 \mathrm{~Hz}$ and $33 \mathrm{~Hz}$, the density of the condensate, and hence the peak speed of sound in the condensate was varied between $1.1 \mathrm{~cm} / \mathrm{s}$ and $0.55 \mathrm{~cm} / \mathrm{s}$.

Impurity atoms were created using a two-photon Raman transition, in which the condensate was exposed to a pair of laser beams [11]. The laser beams had orthogonal linear polarizations, thus driving a Raman transition from the trapped $\left|F=1, m_{F}=-1\right\rangle$ state to the untrapped $\left|F=1, m_{F}=0\right\rangle$ hyperfine ground state. Both beams were derived from a common source, and then passed through two acousto-optic modulators operating with a frequency difference $\omega=\omega_{z}+\hbar q^{2} /(2 M)$, where $\hbar \omega_{z}$ is the Zeeman splitting between the $\left|m_{F}=-1\right\rangle$ and $\left|m_{F}=0\right\rangle$ states in the offset field of the magnetic trap. The momentum transfer from the light field to the $m_{F}=0$ atoms is $\hbar q=2 \hbar k \sin \theta / 2$ where $k$ is the wavevector of the light field and $\theta$ is the angle between the two laser beams. The Raman light field was typically pulsed on for about $10 \mu s$ at an intensity of several $\mathrm{mW} / \mathrm{cm}^{2}$. The fraction of transferred atoms could be varied by changing the light intensity.

Collisions between the impurities and the condensate were analyzed by time-of-flight absorption imaging. For this, the magnetic trap was suddenly switched off $4 \mathrm{~ms}$ after the Raman pulse, by which time the impurity atoms had fully traversed the condensate. After an additional $5 \mathrm{~ms}$, a magnetic field gradient was pulsed on for $30 \mathrm{~ms}$, 
spatially separating the $m_{F}=0$ atoms from the condensate. After a total time-of-flight of typically $60 \mathrm{~ms}$, all atoms were optically pumped into the $\left|F=2, m_{F}=2\right\rangle$ ground state and resonantly imaged on the cycling transition.

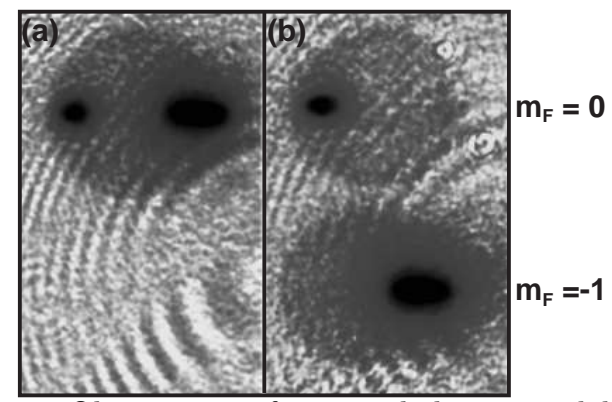

FIG. 1. Observation of $s$-wave halos created by elastic collisions between the condensate and impurity atoms traveling at $6 \mathrm{~cm} / \mathrm{s}$ (to the left in images). (a) Absorption image after $50 \mathrm{~ms}$ of time-of-flight shows the velocity distribution after collisions between the condensate and the outcoupled $m_{F}=0$ atoms. The collisional products are distributed over a sphere in momentum space. (b) same as a), but using a Stern-Gerlach type magnetic field gradient to separate the $m_{F}=0$ atoms (top) from the $m_{F}=-1$ condensate (bottom). The fringes are an imaging artifact. Images are $4.5 \times 7.2 \mathrm{~mm}$

Collisions at ultracold temperatures are in the $s$-wave regime. The products of such collisions between free particles are evenly distributed in momentum space over a spherical shell around the center-of-mass momentum of the collision partners. A time-of-flight picture records the momentum distribution of the released cloud. Thus, collisions between the condensate and the impurities are visible as a circular halo which represents the line-of-sight integrated spherical shell. Fig. 1 shows a typical absorption image of collisions in the free particle regime for impurity atoms with a velocity of $2 \hbar k / M=6 \mathrm{~cm} / \mathrm{s}$, produced by counterpropagating Raman beams.

To probe for superfluidity, we produced impurity atoms at low velocities $(7 \mathrm{~mm} / \mathrm{s})$ by using Raman beams which intersected at an angle of $\approx 14^{\circ}$ and aligned symmetrically about the radial direction, so that the difference vector $\mathbf{q}=\mathbf{k}_{\mathbf{1}}-\mathbf{k}_{\mathbf{2}}$ was directed axially [12]. The trajectory of the impurity atoms was initially in the axial direction, but was soon modified by two forces: a downward gravitational acceleration along a radial direction (into the page in images and hereafter denoted as the $z$ axis), and the radial mean-field repulsion of the $m_{F}=0$ atoms from the $m_{F}=-1$ condensate.

This situation is similar to the previous study of an $\mathrm{rf}$ output coupler by which $m_{F}=0$ atoms were produced at rest [13]. For the rf output coupler, collisions between impurity atoms and the condensate were difficult to detect because the scattered and unscattered atoms were not clearly distinguishable. In this study, the small axial velocity imparted by Raman scattering allowed us to iden- tify products of elastic collisions in time-of-flight images since collisions with the stationary condensate tended to redistribute the impurity atoms toward lower axial velocities. However, the acceleration of the impurity atoms precluded the observation of well-defined collision halos. A time-of-flight analysis of impurity scattering for the case of a low density condensate is shown in Figure 2a. The axial velocity imparted by Raman scattering displaces the unscattered $m_{F}=0$ atoms upward in the image, whereas collisions produce impurity atoms with smaller axial velocities which then appear below the unscattered atoms in the image. In contrast, Figure $2 \mathrm{~b}$ shows a time-of-flight image for the case of a high density condensate, for which the number of collided atoms is greatly diminished, indicating the suppression of impurity collisions due to superfluidity.

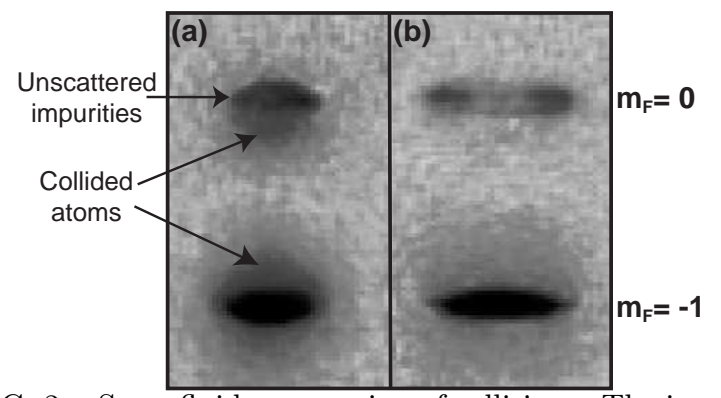

FIG. 2. Superfluid suppression of collisions. The impurity $m_{F}=0$ atoms (top) traveled at $7 \mathrm{~mm} / \mathrm{s}$ along the condensate axis (upward in image) and were separated from the condensate (bottom) by a magnetic field gradient applied during ballistic expansion. (a) Absorption image after $50 \mathrm{~ms}$ of time of flight shows the collisional products as indicated by the arrow. For this image, $v_{g} / c=2.7$ (see text). (b) Similar image as (a) with $v_{g} / c=1.6$. Collisions are suppressed. The outcoupled atoms were distorted by mean-field repulsion. The images are $2.0 \times 4.0 \mathrm{~mm}$.

The number of collided atoms was determined by counting impurity atoms in a region of the time-of-flight image below the unscattered impurity atoms, which also contained Raman outcoupled thermal $m_{F}=0$ atoms. Thus, the number of collided atoms in the counting region was obtained by subtracting the thermal background which was determined by counting a similar sized region above the unscattered impurity atoms where we expect few collision products. This number was doubled to obtain the total number of collided atoms since we expect only about half of the collision products to be in the counting region; the remainder overlapped with the distribution of unscattered impurity atoms.

In studying these collisions, we discovered that the fraction of collided atoms increased with the number of outcoupled impurities (see Fig. 3). According to a perturbative treatment described below, the collision probability should be independent of the number of impurities. If the number of outcoupled atoms is increased, one would 
expect the collision probability to decrease slightly due to the reduction in the condensate density, or to increase slightly because the smaller condensate density implies a smaller critical velocity for dissipation. However, these effects are smaller (10-20\%) than the observed two-fold increase in the collided fraction.

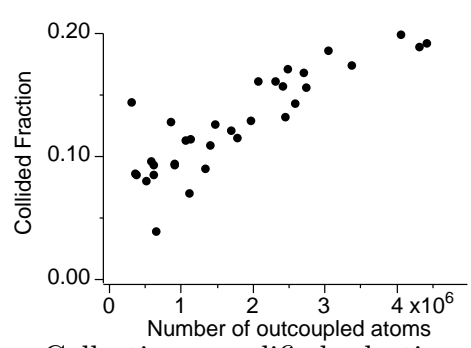

FIG. 3. Collective amplified elastic scattering in a Bose-Einstein condensate. Shown is the fraction of collided atoms vs. the number of outcoupled atoms. For this data, $v_{g} / c=4.9$ and the chemical potential was $1.8 \mathrm{kHz}$.

Rather, this large increase can be explained as a collective self-amplification of atomic scattering, akin to the recently observed superradiant amplification of light scattering from a Bose-Einstein condensate 14. Collisions between impurity atoms and the condensate transfer atoms from a macroscopically occupied initial state to final momentum states which were previously empty. The population in these final states can stimulate further scattering by bosonic enhancement and this effect increases for larger outcoupling. This collisional amplification is not directional, and is similar to the recently observed optical omnidirectional superfluoresence [15]. In contrast, the observation of four-wave mixing of atoms [16] represents the case where collisions were stimulated by a single macroscopically occupied final mode.

Fig. A shows the decrease of collision probability as the velocity of the impurity atoms approached the speed of sound in the condensate. The collision probability was determined by averaging over many iterations of the experiment with the number of outcoupled atoms kept below $10^{6}$, in which case collective effects may be neglected. For our experimental conditions, the impurity velocity was predominantly determined by the gravitational acceleration $g=9.8 \mathrm{~m} / \mathrm{s}^{2}$, which imparted an average velocity of $v_{g}=\sqrt{2 g z_{c}}$ where $z_{c}$ is the Thomas-Fermi radius of the condensate in the $z$-direction. This downward velocity ranged from $17 \mathrm{~mm} / \mathrm{s}$ for tightly confined condensates to $26 \mathrm{~mm} / \mathrm{s}$ for loosely confined condensates, and was larger than the initial $7 \mathrm{~mm} / \mathrm{s}$ velocity imparted by Raman scattering. Thus, the effect of superfluidity on impurity scattering depends primarily on the parameter $\bar{\eta}=v_{g} / c$ which is the ratio of the typical impurity velocity $v_{g}$ to the speed of sound at the center of the condensate $c=\sqrt{\mu / M}$. Experimentally, $\bar{\eta}$ is determined using the radial trapping frequency and the chemical potential $\mu$ which is determined from the expansion of the condensate in the time-of-flight images [17.

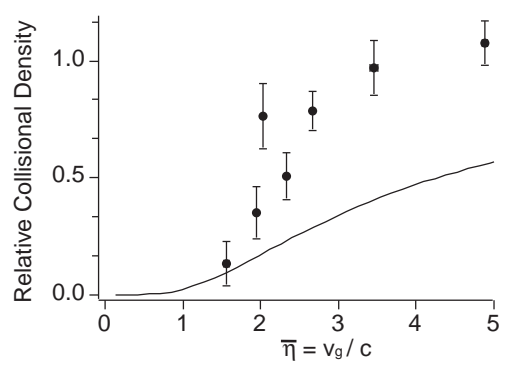

FIG. 4. Onset of superfluid suppression of collisions. Shown is the observed collisional density normalized to the predicted collisonal density $\mathcal{C}_{\infty}$ in the limit of high velocities as the density of the condensate was increased (plotted from right to left). The collisional density is proportional to the collision probability of the outcoupled atoms (see text). The $x$-axis shows $\bar{\eta}=v_{g} / c$ which is a measure of the impurity velocity over the speed of sound. The solid line is the theoretically expected collisional density calculated by a numerical integration of Eq. 1. The error bars represent the statistical uncertainty.

High and low $\bar{\eta}$ correspond to dissipative and nondissipative regimes, respectively. This behavior is apparent in Fig. 2. For the case of loose confinement, the low condensate density ( small $c$ ) and large condensate radius (large $v_{g}$ ) yield a large value for $\bar{\eta} \sim 5$. The effect of collisions is clearly visible with about $20 \%$ of the atoms scattered to lower axial velocities (Fig. 2a). In contrast, for the case of tight confinement, the high condensate density (large $c$ ) and small condensate radius (small $v_{g}$ ) yield a small value for $\bar{\eta} \sim 1.5$, and the collision probability is greatly suppressed due to superfluidity (Fig. 2b).

The predicted cross-section for collisions between an $m_{F}=0$ impurity atom at momentum $\hbar \mathbf{k}$ and a $m_{F}=-1$ condensate of density $n_{0}$ is obtained by calculating the collision rate $\Gamma$ using Fermi's Golden rule [18, 19]:

$$
\begin{aligned}
\Gamma & =n_{0}\left(\frac{\hbar a}{M}\right)^{2} \int d q d \Omega q^{2} S(q) \delta\left(\frac{\hbar \mathbf{k} \cdot \mathbf{q}}{M}-\frac{\hbar q^{2}}{2 M}-\omega_{q}^{B}\right) \\
& =n_{0} \sigma(\eta) v
\end{aligned}
$$

Here, $S(q)=\omega_{q}^{0} / \omega_{q}^{B}$ is the static structure factor of the condensate with $\hbar \omega_{q}^{0}=\hbar^{2} q^{2} / 2 M$ and $\hbar \omega_{q}^{B}=$ $\sqrt{\hbar \omega_{q}^{0}\left(\hbar \omega_{q}^{0}+2 \mu\right)}$ being the energies of a free particle and a Bogoliubov quasiparticle of momentum $q$, respectively. The collision cross section is $\sigma(\eta)=\sigma_{0} F(\eta)$ where $\eta=v / c, v=\hbar k / M$ is the impurity velocity, and $\sigma_{0}=4 \pi a_{0,-1}^{2}$ where $a_{0,-1}=2.75 \mathrm{~nm}$ [20] is the scattering length for $s$-wave collisions between the $\left|m_{F}=0\right\rangle$ and $\left|m_{F}=-1\right\rangle$ states of sodium. For $\eta<1, F(\eta)=0$ and for $\eta>1, F(\eta)=1-1 / \eta^{4}-\log \left(\eta^{4}\right) / \eta^{2}$.

We can approximate our experiment by considering the motion of the $m_{F}=0$ atoms under the gravitational acceleration alone and ignoring the effects of the initial axial velocity and mean-field expulsion [21]. The $m_{F}=0$ 
atoms falling through the condensate experience a collisional density $\mathcal{C}(\eta)=\int d z n(x, y, z) \sigma(\eta)$, where $n(x, y, z)$ is the condensate density, and $\eta$ is determined by the local condensate density and the downward impurity velocity. The collisional density relative to its value at large velocities $\mathcal{C}_{\infty}$ is given by

$$
\frac{\mathcal{C}(\bar{\eta})}{\mathcal{C}_{\infty}} \approx \frac{\int d \mathbf{r} n_{I}(\mathbf{r}) \times \int d z^{\prime} n\left(\mathbf{r}^{\prime}\right) \sigma_{0} F(\eta)}{\int d \mathbf{r} n_{I}(\mathbf{r}) \times \int d z^{\prime} n\left(\mathbf{r}^{\prime}\right) \sigma_{0}}
$$

where we assume that the initial impurity density $n_{I}(\mathbf{r}) \propto$ $n(\mathbf{r})$ [22]. The condensate density in the Thomas-Fermi limit is $n(x, y, z)=n_{0}\left(1-\left(x / x_{c}\right)^{2}-\left(y / y_{c}\right)^{2}-\left(z / z_{c}\right)^{2}\right)$, where $x_{c}=\left(2 \mu / M \omega_{x}^{2}\right)^{1 / 2}$ (similarly for $y_{c}$ and $z_{c}$ ) is the Thomas-Fermi radius, $\omega_{x}$ is the trapping frequency in the $x$ direction, and $\mu$ is the chemical potential. The solid line in Fig. 1 was determined by numerically integrating Eq. 1. To compare the collision probability for the different data points, we divided the observed collided fraction by $\mathcal{C}_{\infty}=(5 / 12) \times n_{0} \sigma_{0} z_{c}$. The observed decrease in the collisional density for small $\bar{\eta}$ (Fig. A) shows the superfluid suppression of collisions. Numerical simulations ruled out the possibility that the observed decrease in collisional density could be caused solely by variations of the path length of particle trajectories due to the mean-field repulsion and the initial velocity.

The measured values in Fig. 4 are systematically larger by a factor of about two than those expected theoretically. This discrepancy is also seen for impurity collisions at velocities of $6 \mathrm{~cm} / \mathrm{s}$ for which superfluidity should play no role. While we cannot presently account for this systematic error, the observation of suppression of collisions due to superfluidity is robust, since it requires only a relative comparison of collision probabilities at different $\bar{\eta}$.

The method presented here can generally be used to study ultra-cold collisions. In this study, we focused on collisions between atoms in different hyperfine states. By driving a Bragg transition [23] instead of a Raman transition, we have also observed collisions between atoms in the same internal state. At a velocity of $6 \mathrm{~cm} / \mathrm{s}$, we found the collision cross section to be $2.1 \pm 0.3$ times larger than in the Raman case, reflecting the exchange term in elastic collisions for identical particles that increases the cross section from $4 \pi a^{2}$ to $8 \pi a^{2}$.

Raman transitions are one way to realize output couplers for atom lasers [11, 13,24,25]. Theoretical treatments of atom lasers have typically considered only the condensate and the outcoupled atoms in a two-mode approximation and ignored the modes accessible by collisions 26]. However, our experiment shows that the outcoupled atoms do not simply pass through the condensate. Rather, they collide and populate modes coupled by atomic scattering [27]; the collisions may even be enhanced by bosonic stimulation. In principle, such collisional losses can be avoided by lowering the density.
However, an alternative route to suppressing collisions is to increase the density until the speed of sound is larger than the velocity of the outcoupled atoms, thus realizing a "superfluid" output coupler.

In conclusion, we have studied collisions between impurity atoms and a Bose-Einstein condensate. Both the observed superfluid suppression of collisions and the collective enhancement are crucial considerations for the future development of intense atom lasers.

We are grateful to D.E. Pritchard for valuable discussions. This work was supported by the ONR, NSF, JSEP, ARO, NASA, and the David and Lucile Packard Foundation. A.P.C. acknowledges additional support from the NSF, A.G. from DAAD, and D.M.S.K. from JSEP and a Robert A. Millikan Postdoctoral Fellowship.

* Present address: Norman Bridge Laboratory of Physics, California Institute of Technology 12-33, Pasadena, CA 91125.

[1] L.D. Landau, J. Phys. (USSR) 5, 71 (1941).

[2] D.R. Tilley and J. Tilley, Superfluidity and Superconductivity (IOP Publishing Ltd., New York, 1990), pp. 41-53.

[3] L. Meyer and F. Reif, Phys. Rev. 123, 727 (1961).

[4] D.R. Allum, P.V.E. McClintock, A. Phillips, and R.M. Bowley, Phil. Trans. R. Soc A 284, 179 (1977).

[5] J. Harms and J.P. Toennies, Phys. Rev. Lett. 83, 344 (1999).

[6] C. Raman et al., Phys. Rev. Lett. 83, 2502 (1999).

[7] M.R. Matthews et al., Phys. Rev. Lett. 83, 2498 (1999).

[8] K.W. Madison, F. Chevy, W. Wohlleben, and J. Dalibard, Phys. Rev. Lett. 84, 806 (2000).

[9] O.M. Marago et al., Phys. Rev. Lett. 84, 2056 (2000).

[10] M.-O. Mewes et al., Phys. Rev. Lett. 77, 416 (1996).

[11] E.W. Hagley et al., Science 283, 1706 (1999).

[12] D.M. Stamper-Kurn et al., Phys. Rev. Lett. 83, 2876 (1999).

[13] M.-O. Mewes et al., Phys. Rev. Lett. 78, 582 (1997).

[14] S. Inouye et al., Science 285, 571 (1999).

[15] A.I. Lvovsky and S.R. Hartmann, Phys. Rev. Lett. 82, 4420 (1999).

[16] L. Deng et al., Nature 398, 218 (1999).

[17] W. Ketterle, D.S. Durfee, and D.M. Stamper-Kurn, in Bose-Einstein condensation in atomic gases, Proceedings of the International School of Physics Enrico Fermi, Course CXL, edited by M. Inguscio, S. Stringari, and C.E. Wieman (IOS Press, Amsterdam, 1999), pp. 67176 .

[18] E. Timmermans and R. Côté, Phys. Rev. Lett. 80, 3419 (1998).

[19] Z. Idziaszek, K. Rzazewski, and M. Wilkens, J. Phys. B 32, L205 (1999).

[20] E. Tiesinga et al., J. Res. Natl. Inst. Stand. Technol. 101, 505 (1996).

[21] A numerical simulation showed that including these ef- 
fects changes the collisonal density by less than $20 \%$.

[22] The Raman transition strength is uniform over the entire condensate since the frequency broadening due to finite pulse-length $(16 \mathrm{kHz})$ was larger than the broadening due to mean-field $(\sim 2 \mathrm{kHz})$.

[23] J. Stenger et al., Phys. Rev. Lett. 82, 4569 (1999).

[24] I. Bloch, T.W. Hänsch, and T. Esslinger, Phys. Rev. Lett. 82, 3008 (1998).

[25] B.P. Anderson and M.A. Kasevich, Science 282, 1686 (1998).

[26] See for example: R.J. Ballagh, K. Burnett, and T.F. Scott, Phys. Rev. Lett. 78, 1607 (1997); Y.B. Band, P.S. Julienne, and M. Trippenbach, Phys. Rev. A 59, 3823 (1999).

[27] Motivated by our results, collisions were recently included in a theoretical treatment of interaction between condensates: Y.B. Band, M. Trippenbach, J.P. Burke Jr., and P.S. Julienne, preprint, submitted. 